\title{
Commentary on long-term efficacy and patient satisfaction of Le Fort colpocleisis for the treatment of severe pelvic organ prolapse
}

\author{
Kaitlin Renkosiak ${ }^{1} \cdot$ Alexandra Dubinskaya $^{1}$ (I) \\ Received: 23 June 2020 / Accepted: 6 July 2020 / Published online: 20 July 2020 \\ (C) The International Urogynecological Association 2020
}

The incidence of pelvic organ prolapse continues to rise secondary to an aging female population, leading to associated decrease in quality of life among affected women. While there are various surgical interventions aimed at correcting prolapse, each procedure carries different rates of efficacy and patient satisfaction. The Le Fort procedure can be used to correct prolapse but is classically restricted to women no longer desiring vaginal intercourse. Wang et al. in their study strived to quantify both efficacy and patient satisfaction associated with Le Fort colpocleisis.

In this retrospective study, 317 patients who underwent Le Fort colpocleisis between January 2007 to August 2018 were analyzed at a single hospital. Approximately two-thirds of patients (208) were available for either outpatient or telephone follow-up. Follow-up was aimed at determining postoperative anatomic recurrence, new-onset symptoms related to urinary or intestinal complaints, and patient satisfaction using a Patient Global Impression of Improvement scale. Using ttests and chi-square tests, the authors demonstrated follow- up $>3$ years in nearly $70 \%$ of their patients with no reported anatomic recurrence in the study. Moreover, patient satisfaction scores using PGI-I demonstrated that $99.5 \%$ of women felt their symptoms were either "improved" or "very much improved."

While the study noted no anatomic recurrence of prolapse, only $14 \%$ of women received an outpatient physical examination to clearly document lack of recurrence, thus limiting the ability to objectively quantify efficacy. Despite this limitation, the authors demonstrated that colpocleisis with or without concomitant hysterectomy remains a procedure with high patient satisfaction and low occurrence of regret $(0.96 \%)$ for women with severe pelvic organ prolapse who no longer desire vaginal penetrative intercourse.

Publisher's note Springer Nature remains neutral with regard to jurisdictional claims in published maps and institutional affiliations.

Alexandra Dubinskaya

a.dubinsky.md@gmail.com

1 St Francis Hospital and Medical Center, Hartford, CT, USA 